\title{
AMPK activation restores ischemic post-conditioning cardioprotection in STZ-induced type 1 diabetic rats: Role of autophagy
}

\author{
BIN ZHOU, YAN LENG, SHAO-QING LEI and ZHONG-YUAN XIA \\ Department of Anesthesiology, Renmin Hospital of Wuhan University, Wuhan, Hubei 430060, P.R. China
}

Received May 31, 2016; Accepted April 7, 2017

DOI: $10.3892 / \mathrm{mmr} .2017 .7033$

\begin{abstract}
Although the mechanism remains unclear, ischemic post-conditioning (IPO) is a promising approach to combat myocardial ischemia reperfusion (IR) injury; however, it has been proven ineffective in diabetes. The present study aimed to identify whether hyperglycemia-induced AMP-activated protein kinase (AMPK) inhibition contributes to the ineffectiveness of IPO via autophagy attenuation in diabetic hearts. Diabetic and non-diabetic rats were subjected to myocardial IR and/or IPO with/without treatment with the AMPK activator A-769662 and/or autophagy inhibitor 3-methyladenine (3-MA). Rat cardiomyocyte H9c2 cells were pretreated with A-769662 and/or 3-MA, and subjected to hypoxia reoxygenation (HR) or hypoxia post-conditioning (HPO). The degree of injury to the myocardium/cells, oxidative stress, AMPK/mammalian target of rapamycin (mTOR) signaling and autophagy status were analyzed. In diabetic rats the myocardial infarct size, and creatine kinase-MB and malondialdehyde release, were increased compared with non-diabetic rats, concomitant with increased cardiac dysfunction and decreased cardiac superoxide dismutase activity, AMPK phosphorylation and autophagy following IR. IPO attenuated myocardial infarct size, increased AMPK phosphorylation and enhanced autophagy in non-diabetic animals. A-769662 $(6.0 \mathrm{mg} / \mathrm{kg})$ restored IPO cardioprotection in diabetic rats. In vitro, HPO combined with A-769662 decreased HR injury in H9c2 cells exposed to high glucose, as evidenced by decreased lactic dehydrogenase expression and oxidative stress, accompanied by increased cell viability and autophagy. The A-769662-mediated restoration of IPO/HPO cardioprotection was completely reversed by treatment with the autophagy inhibitor 3-MA. In conclusion, AMPK inhibition, by decreasing autophagy, may be a
\end{abstract}

Correspondence to: Dr Zhong-Yuan Xia, Department of Anesthesiology, Renmin Hospital of Wuhan University, 238 Jiefang Road, Wuhan, Hubei 430060, P.R. China

E-mail: xiazhongyuanmz@aliyun.com

Key words: AMP-activated protein kinase, serine/threonine protein kinase mTOR, autophagy, ischemic post-conditioning, myocardium, diabetes mechanism through which diabetic hearts are rendered unresponsive to IPO cardioprotection.

\section{Introduction}

The prevalence of diabetes has increased worldwide. Previous studies have demonstrated that patients with diabetes are more vulnerable to myocardial ischemia reperfusion (IR) injury, and the risk of post-myocardial infarction death is increased by $200-400 \%$ in patients with diabetes compared with non-diabetic individuals (1). Ischemic post-conditioning (IPO), administered at the onset of reperfusion, has been demonstrated to be an effective method to combat myocardial IR injury $(2,3)$. IPO may be a more promising approach compared with ischemic preconditioning (IPC) due to the difficulties associated with predicting the onset of myocardial ischemia in clinical practice. However, previous studies have demonstrated that diabetic hearts were unresponsive to IPO, and the underlying mechanisms remain unclear (4-7).

AMP-activated protein kinase (AMPK), an evolutionarily conserved serine/threonine kinase, is a principal regulator of adenosine 5'-triphosphate homeostasis and energy metabolism in the body (8). AMPK serves a role in cell survival under stress conditions, including oxidative stress, starvation, ischemia and hypoxia (9-11). The beneficial effects of AMPK activation are mediated by maintaining the homeostasis of reduced nicotinamide adenine dinucleotide phosphate (NADPH), phosphorylating the tuberous sclerosis complex to inhibit mammalian target of rapamycin (mTOR), thereby promoting cytoprotective autophagy though direct (serine/threonine protein kinase ULK1 phosphorylation) or indirect (mTOR inhibition) mechanisms $(10,12)$. Autophagy is a conserved intracellular self-digestion process for long-lived cytoplasmic proteins, organelles and macromolecules, and is essential for maintaining cellular homeostasis under normal conditions and affording protective responses to adverse conditions (13).

Previous studies have observed that AMPK activation is able to induce autophagy and, subsequently, provide protective effects against IR injury in heart (14), brain (15), liver (16), kidney (17) and muscular tissues (18). IPC and IPO have been demonstrated to combat IR injury by triggering AMPK-regulated autophagy $(15,19)$. In the diabetic myocardium, AMPK was observed to be inhibited in combination with decreased cardiac autophagy, and further studies 
demonstrated that cardiac function was improved in diabetes by promoting AMPK-regulated autophagy (20). These previous experimental data suggested that AMPK-regulated autophagy may serve a role in protecting the myocardium against IR injury and hyperglycemic insult. However, whether AMPK-regulated autophagy is associated with the pathophysiological process of myocardial IR injury in diabetes, and its underlying mechanisms, remains to be elucidated.

The aims of the present study were to investigate whether hyperglycemia-induced AMPK inhibition is responsible for the ineffectiveness of IPO by impairing autophagy in diabetic hearts, and if so, whether activation of AMPK is able to restore the sensitivity of diabetic hearts to IPO-induced cardioprotection through autophagy activation.

\section{Materials and methods}

Experimental animals. A total of 120 male Sprague-Dawley rats of specific pathogen-free level, weighing 250 $10 \mathrm{~g}$ (age, 6-8 weeks) were provided by Hunan SLAC JD Laboratory Animal Co., Ltd. (Hunan, China). All rats were housed at $24^{\circ} \mathrm{C}$, with a fixed light/ dark cycle (12 h light/12 h dark) and with ad libitum access to food and water. All of the experimental protocols were in accordance with the principles of Animal Care of Wuhan University (Wuhan, China), and approved by the Committee for the Use of Live Animals in Teaching and Research. Diabetic rats were induced by a single intraperitoneal (i.p.) injection of streptozotocin $(60 \mathrm{mg} / \mathrm{kg}$; Sigma-Aldrich; Merck KGaA, Darmstadt, Germany) as previously described, and rats exhibiting hyperglycemia (blood glucose $\geq 16.7 \mathrm{mmol} / \mathrm{l}$ ) were considered to be diabetic $(4,5)$. The body weight, blood glucose and food and water intake of all rats were observed and recorded.

Myocardial IR injury model. A well-established myocardial IR injury model was used in the present study (4). All rats were anesthetized (sodium pentobarbital; $50 \mathrm{mg} / \mathrm{kg}$ i.p.; Sigma-Aldrich; Merck KGaA) with tracheotomy and ventilation. The IR injury model was achieved by occluding the left anterior descending artery for $30 \mathrm{~min}$ followed by $120 \mathrm{~min}$ of reperfusion. IPO was established by 3 cycles of $10 \mathrm{sec}$ of reperfusion and ischemia at the onset of reperfusion. Sham-operated rats were subjected to the same surgical procedures without ligation. Ischemia was confirmed by elevation of the ST segment with limb lead II and discoloration of the ischemic zone.

Experimental protocols. A total of 8 weeks subsequent to the onset of diabetes, diabetic (D) and age-matched non-diabetic (N) rats were randomly divided into 10 groups ( $n=12 /$ group) as follows: 1, N+sham (S); 2, N+IR; 3, N+IPO; 4, D+S; 5, D+IR; 6, D+IPO; 7, D+IR+A-769662; 8, D+IPO+A-769662; 9, D+IPO+3-MA+A-769662; and 10, D+IR+3-MA. A-769662 (6 mg/kg; catalogue no. S2697) (21) and 3-MA (catalogue no. S2767; $15 \mathrm{mg} / \mathrm{kg}$ ) (both from Selleck Chemicals, Houston, TX, USA) (22) were given as i.p. injections $30 \mathrm{~min}$ prior to myocardial ischemia.

Cardiac function assessment. Invasive hemodynamic monitoring was performed to evaluate cardiac function.
Left ventricular systolic pressure (LVSP), maximal rates of increase and decrease in LVSP $\left( \pm \mathrm{dP} / \mathrm{dt}_{\max }\right)$, and heart rate (HR) were intermittently monitored using an electrophysiolograph (MH150; BioPAC Systems, Inc., Goleta, CA, USA) and the data were analyzed using AcqKnowledge software (version 5.0; BioPAC Systems, Inc.).

Infarct size determination. Myocardial infarct size was measured using 3\% Evans blue dye and 1\% 2,3,5-triphenyltetrazolium chloride (both from Sigma-Aldrich; Merck KGaA) staining, and scanning (v30; Seiko Epson Corporation, Nagano, Japan) and image analysis using Image-Pro Plus software (version 3.0, Media Cybernetics, Inc., Rockville, MD, USA), as described previously (4). The risk areas were stained red, while the infarct areas remained pale.

Creatine kinase- $M B(C K-M B)$ assay. Blood samples were centrifuged $\left(1,200 \times \mathrm{g}\right.$ for $10 \mathrm{~min}$ at $\left.4^{\circ} \mathrm{C}\right)$, and the serum was collected to measure CK-MB using commercial kits (catalogue no. 1327c; Elabscience Biotechnology Co., Ltd., Wuhan, China), according to the manufacturer's protocol.

Oxidative stress detection. Myocardial tissue and H9c2 cells were homogenized and centrifuged $(2,400 \mathrm{x} \mathrm{g}$ for $15 \mathrm{~min}$ at $4^{\circ} \mathrm{C}$ ) to obtain the supernatants. The activity of superoxide dismutase (SOD) was detected using a SOD assay kit, which employed the hydroxylamine method (catalogue no. A001-1; Nanjing Jiancheng Bioengineering Institute, Nanjing, China). The expression of malondialdehyde (MDA) was determined using an ELISA assay kit (catalogue no. 0060c; Elabscience Biotechnology Co., Ltd.), according to the manufacturer's protocol.

Electron microscopy. Observation of the number of autophagosomes under a transmission electron microscope (TEM) is a direct qualitative measure of autophagy (23). Ischemic heart tissue samples of $\sim 1 \mathrm{~mm}^{3}$ were removed and pre-fixed in a solution of $2.5 \%$ glutaraldehyde at $4^{\circ} \mathrm{C}$ for $24 \mathrm{~h}$, and subsequently post-fixed in $1 \% \mathrm{OsO}_{4}$ at $4^{\circ} \mathrm{C}$ for $30 \mathrm{~min}$, dehydrated in an ascending series of alcohol, and embedded in epoxy resin. The slide was stained by uranyl acetate and lead citrate at $4^{\circ} \mathrm{C}$ for 0.5-1 h, and observed under a TEM (HT7700; Hitachi, Ltd., Tokyo, Japan).

Study in $\mathrm{H} 9 \mathrm{c} 2$ cell lines. Rat cardiomyocyte-derived H9c2 cells (American Type Culture Collection, Manassas, VA, USA) were maintained in Dulbecco's modified Eagle's medium (Gibco; Thermo Fisher Scientific, Inc., Waltham, MA, USA) containing 10\% fetal bovine serum (Gibco; Thermo Fisher Scientific, Inc.) and $100 \mu \mathrm{g} / \mathrm{ml}$ penicillin/streptomycin in an atmosphere containing $5 \% \mathrm{CO}_{2}$ at $37^{\circ} \mathrm{C}$. The cells were randomly divided into 10 groups: 1 , low glucose $(5.5 \mathrm{mM})$ medium (LG); 2, LG+hypoxia/reoxygenation (HR); 3, LG+hypoxia post-conditioning (HPO); 4, high glucose (30 mM) medium (HG); 5, HG+HR; 6, $\mathrm{HG}+\mathrm{HPO} ; 7, \mathrm{HG}+\mathrm{HR}+\mathrm{A}-769662 ; 8, \mathrm{HG}+\mathrm{HPO}+\mathrm{A}-769662 ; 9$, $\mathrm{HG}+\mathrm{HPO}+\mathrm{A}-769662+3-\mathrm{MA} ; 10, \mathrm{HG}+\mathrm{HR}+3-\mathrm{MA}$. A-769662 $(100 \mathrm{mM})$ and $3-\mathrm{MA}(10 \mathrm{nM})(24)$ was given $1 \mathrm{~h}$ prior to hypoxia, and the cells underwent $4 \mathrm{~h}$ of hypoxia followed by $2 \mathrm{~h}$ of reoxygenation. HPO was performed by 3 cycles 
of 5 min reoxygenation and hypoxia. Hypoxic conditions were obtained using a gas incubator $\left(5 \% \mathrm{CO}_{2}\right.$ and $\left.95 \% \mathrm{~N}_{2}\right)$. Each experiment was performed $\geq 3$ times independently in triplicate. Cells and supernatants were collected for further analysis.

Cell viability and lactate dehydrogenase ( $L D H)$ release assay. Cell viability was determined using a Cell Counting Kit-8 (CCK-8) assay kit at a wavelength of $450 \mathrm{~nm}$ (catalogue no. 04-11; Dojindo Molecular Technologies, Inc., Kumamoto, Japan), and LDH was measured using a cytotoxicity assay kit a wavelength of $490 \mathrm{~nm}$ (catalogue no. 0218c; Elabscience Biotechnology Co., Ltd.), according to the manufacturer's protocols.

Western blot analysis. Western blotting was performed as described previously (4). Tissues or cells were homogenized with radioimmunoprecipitation assay lysis buffer. Equivalent proteins were separated using SDS-PAGE on a 5-15\% gel and electro-transferred onto a polyvinylidene fluoride membrane. The membranes were incubated with anti-GAPDH (catalogue no. 2118), anti-microtubule associated protein 1 light chain $3 \beta / \alpha$ (LC3B/A, catalogue no. 12741), anti-nuclear pore glycoprotein p62 (p62, catalogue no. 5114), anti-mTOR (catalogue no. 2983), anti-phosphorylated (p) mTOR (ser2448, catalogue no. 5536) (all from Cell Signaling Technology, Inc., Danvers, MA, USA), AMPK $\alpha$ (catalogue no. sc25792) and p-AMPK $\alpha$ (Thr172, catalogue no. sc101630) (both from Santa Cruz Biotechnology, Inc., Dallas, TX, USA) primary antibodies (1:500-1,000 dilution) overnight at $4^{\circ} \mathrm{C}$, followed by Alexa Fluor secondary antibody (1:10,000 dilution, catalogue no. A-21210; Thermo Fisher Scientific, Inc.) for $1 \mathrm{~h}$ at room temperature. Signals were detected using an Odyssey fluorescence imaging scanner and quantified using Odyssey software v3.0.29 (both from LI-COR Biosciences, Lincoln, NE, USA).

Statistical analysis. Rats and H9c2 cell culture dishes were randomly assigned to treated or control groups. Western blot analysis was conducted blindly, with samples separated into numbered groups at random. Data are presented as the mean \pm standard deviation. An unpaired Student's t-test was used to detect the differences in characteristics between non-diabetic and diabetic rats. Two-way repeated-measures analysis of variance (ANOVA) followed by Bonferroni's post-hoc test was used to analyze the differences in left ventricular function data between the groups. All other data were evaluated using one-way ANOVA followed by Bonferroni's post-hoc test. Analysis was performed using Prism software (version 5.0.7; GraphPad Software, Inc., La Jolla, CA, USA). $\mathrm{P}<0.05$ was considered to indicate a statistically significant difference.

\section{Results}

Characteristics of experimental diabetic rats. No significant difference was observed in body weight and blood glucose prior to diabetes induction. Following 8 weeks of STZ-induced diabetes, the rats were characterized by a decreased body weight, increased blood glucose, and increased food and water intake compared with the age-matched non-diabetic rats (data not presented).

IPO provides cardioprotection in non-diabetic animals. No significant difference was observed in the area at risk as a percentage of the left ventricle (AAR/LV) in all groups (data not presented). As presented in Fig. 1A, diabetic rats exhibited increased myocardial infarct size compared with non-diabetic rats, following IR insult. IPO significantly decreased the infarct size in non-diabetic rats, and not in diabetic rats. Biochemical markers of myocardial injury and oxidative stress were additionally examined. Diabetic hearts exhibited an increased level of MDA and decreased activity of SOD. Compared with non-diabetes, IR significantly increased CK-MB and MDA level and decreased SOD activity in diabetes. IPO caused a significant reversion in non-diabetes, and not in diabetes (Fig. 1B-D).

Effects of IPO on myocardial autophagy status, and AMPK and mTOR expression in diabetes and non-diabetes. In the present study, myocardial autophagy status, and AMPK and mTOR expression and phosphorylation, were observed following IR injury or IPO treatment. As presented in Fig. 2A-D, compared with non-diabetes, a decreased autophagosome number and LC3B/A ratio, in combination with increased p62 expression, were observed in diabetes. IR insult significantly increased the autophagosome number and LC3B/A ratio, and decreased p62 expression, in non-diabetes; these alterations were further increased by IPO. However, IR and IPO did not significantly alter myocardial autophagosome number, LC3B/A ratio and p62 expression in diabetic hearts. No significant difference was detected in the total expression of AMPK and mTOR among all the groups (data not presented). As presented in Fig. 2E and F, in non-diabetes, IR increased AMPK phosphorylation and decreased mTOR phosphorylation, which was further increased by IPO. Compared with non-diabetes, a decrease in phosphorylated AMPK with an increase in phosphorylated mTOR were detected in the diabetic myocardium. IR and IPO were observed to increase AMPK phosphorylation and decrease mTOR phosphorylation.

AMPK activation by A-769662 restores the protective effects of IPO in diabetic hearts. The present study investigated whether A-769662 is able to restore IPO cardioprotection in diabetes, and whether these effects may be affected by the autophagy inhibitor 3-MA. As presented in Fig. 1, administration of A-769662 alone failed to decrease myocardium infarct size, and CK-MB and MDA level, and to increase SOD activity. By contrast, A-769662 with IPO significantly decreased the infarct size, decreased CK-MB and MDA expression, and elevated SOD activity. All of these effects were reversed by treatment with the autophagy inhibitor 3-MA, although 3-MA alone did not influence the infarct size, CK-MB and MDA release, and SOD activity in diabetic rats following IR insult. Hemodynamic parameters reflecting left ventricular function were analyzed in the present study. Diabetic rats exhibited markedly decreased HR, LVSP, $+\mathrm{dP} / \mathrm{dt}$ and $-\mathrm{dP} / \mathrm{dt}$ compared with age-matched non-diabetic animals at baseline (data not presented). As presented in Table I, all of the hemodynamic parameters were decreased in the diabetic and non-diabetic 
Table I. Hemodynamic parameters as markers of left ventricular function following $2 \mathrm{~h}$ reperfusion.

\begin{tabular}{|c|c|c|c|c|}
\hline Groups & HR, bpm & LVSP, $\mathrm{mmHg}$ & $+\mathrm{dP} / \mathrm{dt}, \mathrm{mmHg} / \mathrm{s}$ & $-\mathrm{dP} / \mathrm{dt}, \mathrm{mmHg} / \mathrm{s}$ \\
\hline $\mathrm{N}+\mathrm{S}$ & $361 \pm 14$ & $122 \pm 5$ & $6362 \pm 130$ & $4872 \pm 130$ \\
\hline $\mathrm{N}+\mathrm{IR}$ & $280 \pm 8^{a}$ & $89 \pm 4^{\mathrm{a}}$ & $4416 \pm 151^{\mathrm{a}}$ & $3237 \pm 154^{\mathrm{a}}$ \\
\hline $\mathrm{N}+\mathrm{IPO}$ & $338 \pm 14^{\mathrm{b}}$ & $112 \pm 4^{b}$ & $5368 \pm 176^{c}$ & $4465 \pm 135^{\mathrm{c}}$ \\
\hline $\mathrm{D}+\mathrm{S}$ & $288 \pm 10^{\mathrm{a}}$ & $100 \pm 4^{\mathrm{a}}$ & $4641 \pm 166^{a}$ & $3537 \pm 163^{a}$ \\
\hline $\mathrm{D}+\mathrm{IR}$ & $169 \pm 9^{c}$ & $53 \pm 4^{c}$ & $2781 \pm 158^{c}$ & $2225 \pm 112^{c}$ \\
\hline $\mathrm{D}+\mathrm{IR}+\mathrm{A}$ & $178 \pm 8$ & $57 \pm 4$ & $2841 \pm 184$ & $2349 \pm 248$ \\
\hline $\mathrm{D}+\mathrm{IPO}$ & $201 \pm 12$ & $67 \pm 5$ & $2628 \pm 168$ & $2506 \pm 129$ \\
\hline $\mathrm{D}+\mathrm{IPO}+\mathrm{A}$ & $269 \pm 12^{\mathrm{d}}$ & $91 \pm 4^{\mathrm{d}}$ & $4358 \pm 149^{d}$ & $3212 \pm 143^{\mathrm{d}}$ \\
\hline $\mathrm{D}+\mathrm{IPO}+\mathrm{A}+3-\mathrm{MA}$ & $205 \pm 9^{\mathrm{e}}$ & $62 \pm 6^{\mathrm{e}}$ & $2561 \pm 143^{\mathrm{e}}$ & $2429 \pm 176^{\mathrm{e}}$ \\
\hline
\end{tabular}

Values are presented as the mean \pm standard deviation (n=8-12/group). ${ }^{\mathrm{a}} \mathrm{P}<0.01 \mathrm{vs}$. $\mathrm{N}+\mathrm{S}$ group; ${ }^{\mathrm{b}} \mathrm{P}<0.05,{ }^{\mathrm{C}} \mathrm{P}<0.01 \mathrm{vs}$. $\mathrm{N}+\mathrm{IR}$ group; ${ }^{\mathrm{d}} \mathrm{P}<0.01 \mathrm{vs}$. $\mathrm{D}+\mathrm{IR}$ group; ${ }^{\mathrm{e}} \mathrm{P}<0.01$ vs. D+IPO+A group. N, non-diabetes; D, diabetes; $\mathrm{S}$, sham; IR, ischemia reperfusion; IPO, ischemic post-conditioning; A, A-769662; HR, heart rate; LVSP, left ventricular systolic pressure; $\mathrm{dP} / \mathrm{dt}$, change in LVSP.
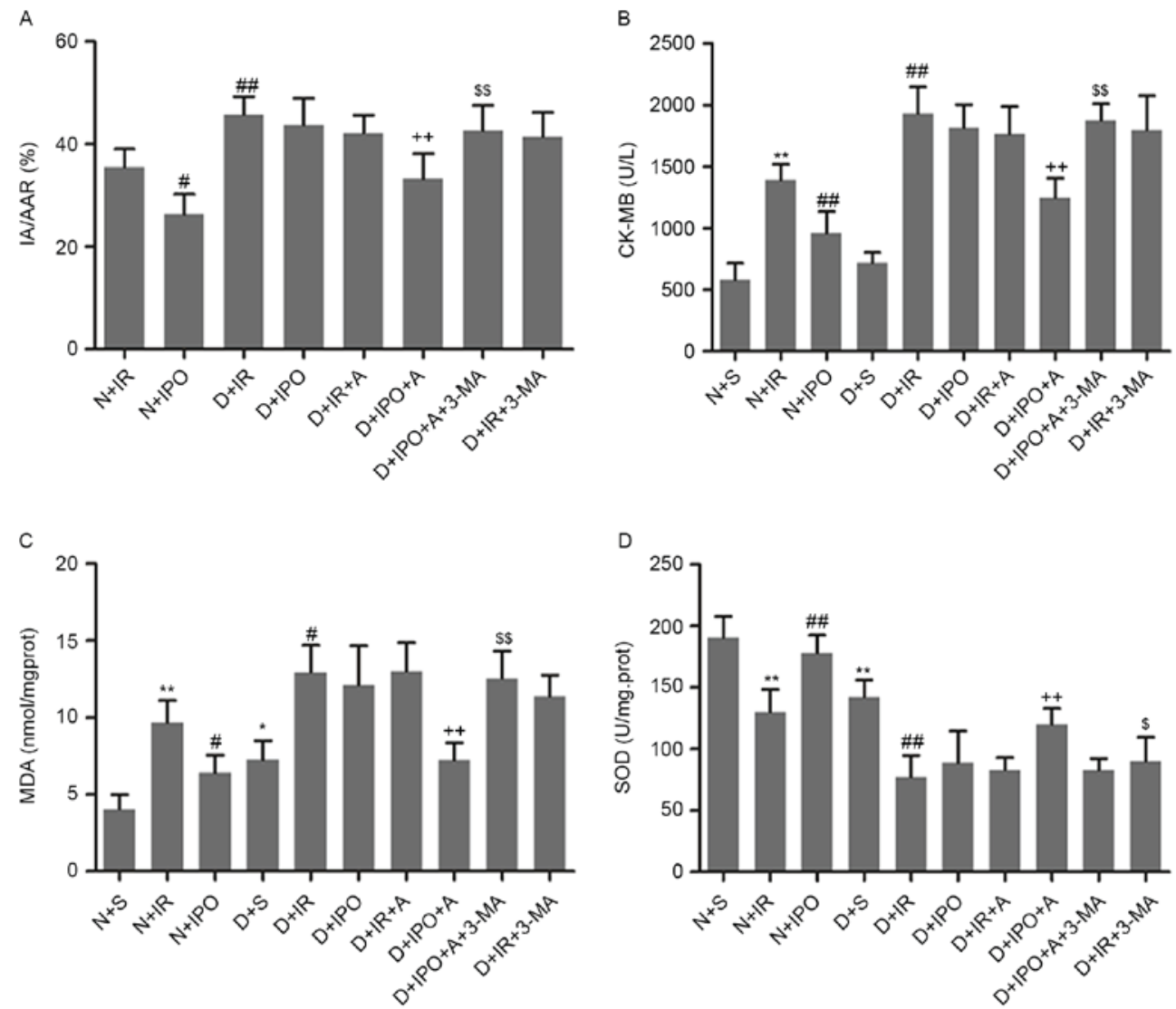

Figure 1. Effects of IPO on myocardial infarct size and biomarkers following $30 \mathrm{~min}$ ischemia followed by $2 \mathrm{~h}$ reperfusion, in non-diabetic and diabetic rats. (A) Percentage of area at risk vs left ventricle. Biomarkers of the degree of injury were (B) CK-MB (C) MDA and (D) SOD. $n=6 /$ group. ${ }^{* *} \mathrm{P}<0.01$ vs. N+S group; ${ }^{\#} \mathrm{P}<0.05$ and ${ }^{\# \#} \mathrm{P}<0.01$ vs. N+IR group; ${ }^{++} \mathrm{P}<0.01$ vs. $\mathrm{D}+\mathrm{S}$ group; ${ }^{\mathrm{P}} \mathrm{P}<0.05$ and ${ }^{\$ \$} \mathrm{P}<0.01$ vs. D+IPO+A group. N, non-diabetes; D, diabetes; $\mathrm{S}$, sham; IR, ischemia reperfusion; IPO, ischemic post-conditioning; A, A-769662; IA/AAR, infarct area/area at risk; CK-MB, creatine kinase MB; MDA, malondialdehyde; SOD, superoxide dismutase.

groups following $2 \mathrm{~h}$ reperfusion. IPO significantly increased the level of HR, LVSP, $+\mathrm{dP} / \mathrm{dt}$ and $-\mathrm{dP} / \mathrm{dt}$ in non-diabetic animals. Treatment with A-769662 alone did not alter the hemodynamic parameters, compared with the untreated group. However, A-769662 treatment with IPO increased the levels of $\mathrm{HR}, \mathrm{LVSP},+\mathrm{dP} / \mathrm{dt}$ and $-\mathrm{dP} / \mathrm{dt}$ in diabetic rats. Notably, all of the alterations in hemodynamic parameters were reversed by treatment with 3-MA.

Effects of AMPK activation on myocardial autophagy and the AMPK-mTOR signaling pathway in diabetes. In order to investigate the underlying mechanisms, the present study 
A

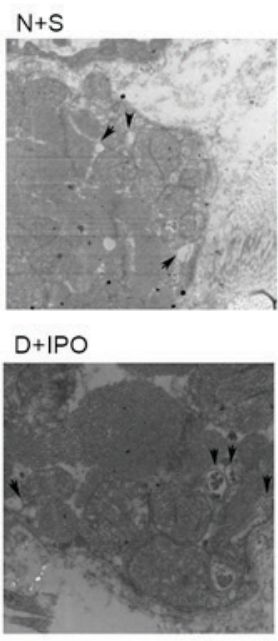

$\mathrm{N}+\mathrm{IR}$

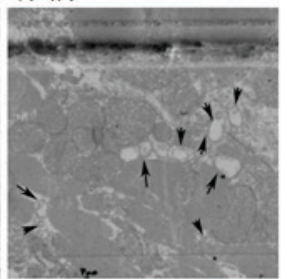

$D+I R+A$

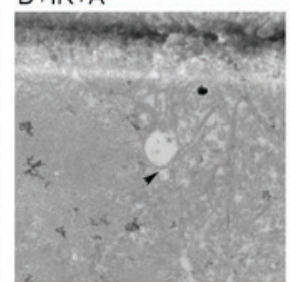

B

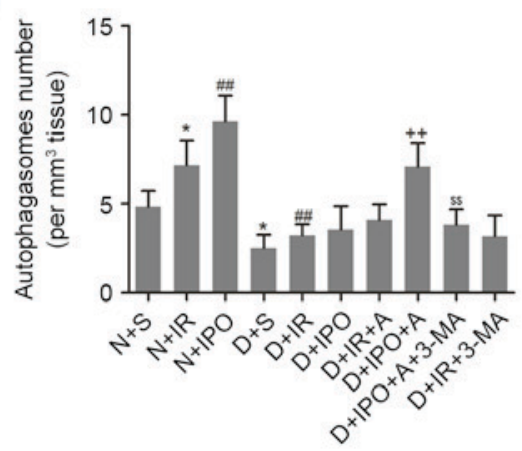

$\mathrm{N}+\mathrm{IPO}$

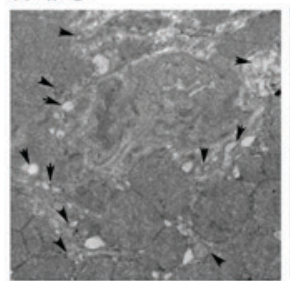

$D+I P O+A$

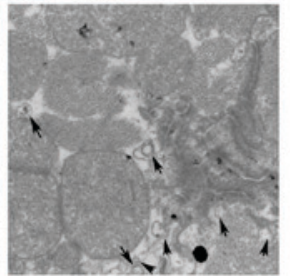

C

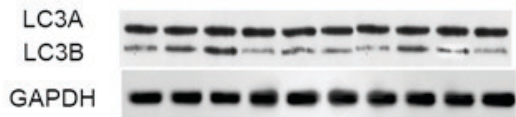

GAPDH

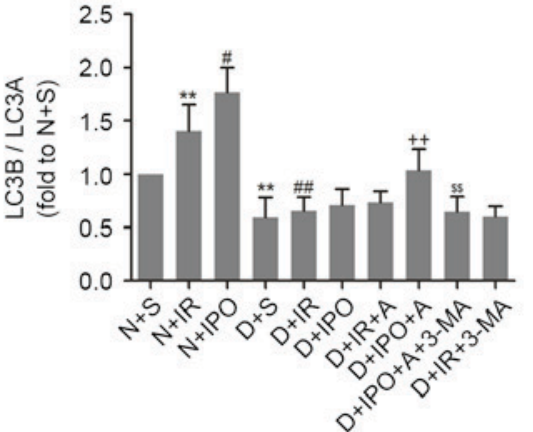

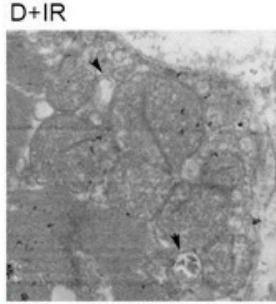

$D+I R+3-M A$
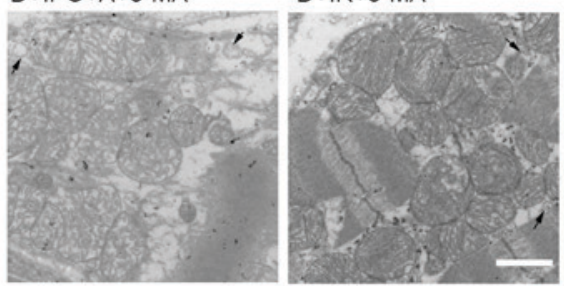

D
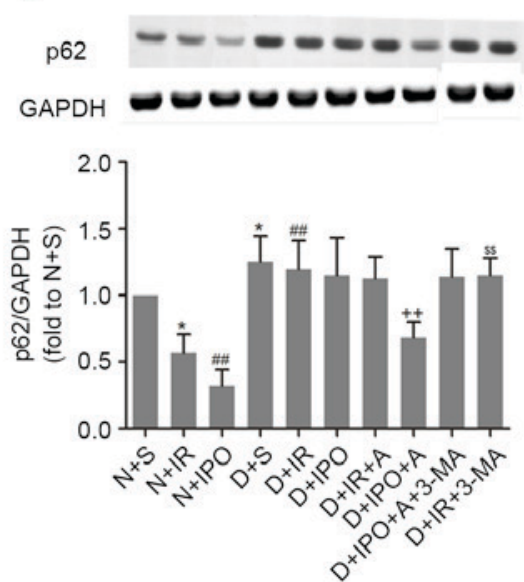

E
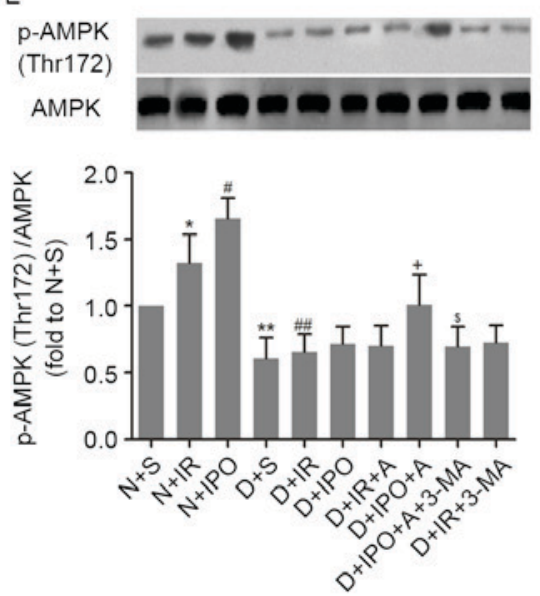

$\mathrm{F}$
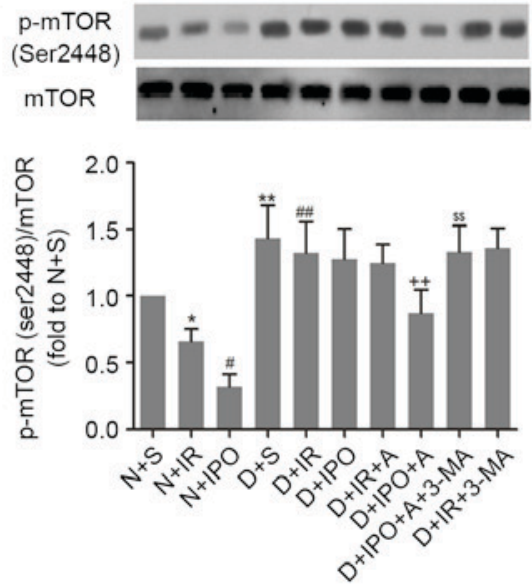

Figure 2. Effects of IPO with or without A on myocardial autophagy and the AMPK/mTOR signaling pathway in non-diabetic and diabetic rats, with 30 min ischemia followed by $2 \mathrm{~h}$ reperfusion. (A) Myocardial transmission electron microscopy analysis. (B) Autophagosome number. (C) Ratio of LC3B/A. (D) Expression of p62. (E) AMPK phosphorylation. (F) mTOR phosphorylation. Scale bar $=1 \mu \mathrm{m}$. $\mathrm{n}=6 / \mathrm{group}$. ${ }^{*} \mathrm{P}<0.05$ and ${ }^{* * *} \mathrm{P}<0.01$ vs. N+S group; ${ }^{\#} \mathrm{P}<0.05$ and ${ }^{\# \#} \mathrm{P}<0.01$ vs. N+IR group; ${ }^{+} \mathrm{P}<0.05$ and ${ }^{++} \mathrm{P}<0.01$ vs. D+IR group; and ${ }^{\$} \mathrm{P}<0.05$ and ${ }^{\$} \mathrm{P}<0.01$ vs. D+IPO+A group. N, non-diabetes; D, diabetes; $\mathrm{S}$, sham; IR, ischemia reperfusion; IPO, ischemic post-conditioning; A, A-769662; LC3, microtubule associated protein 1 light chain 3; AMPK, 5'-AMP-activated protein kinase catalytic subunit $\alpha-1$; mTOR, mammalian target of rapamycin; p62, nuclear pore glycoprotein p62; p, phosphorylated.

analyzed the effects of A-769662 on myocardial autophagy status and the AMPK/mTOR signaling pathway. As presented in Fig. 2, A-769662 administration or IPO alone did not affect myocardial autophagy status and the AMPK/mTOR signaling pathway in diabetes. However, A-769662 combined with IPO increased the autophagosome number, $\mathrm{LC} 3 \mathrm{~B} / \mathrm{A}$ ratio 

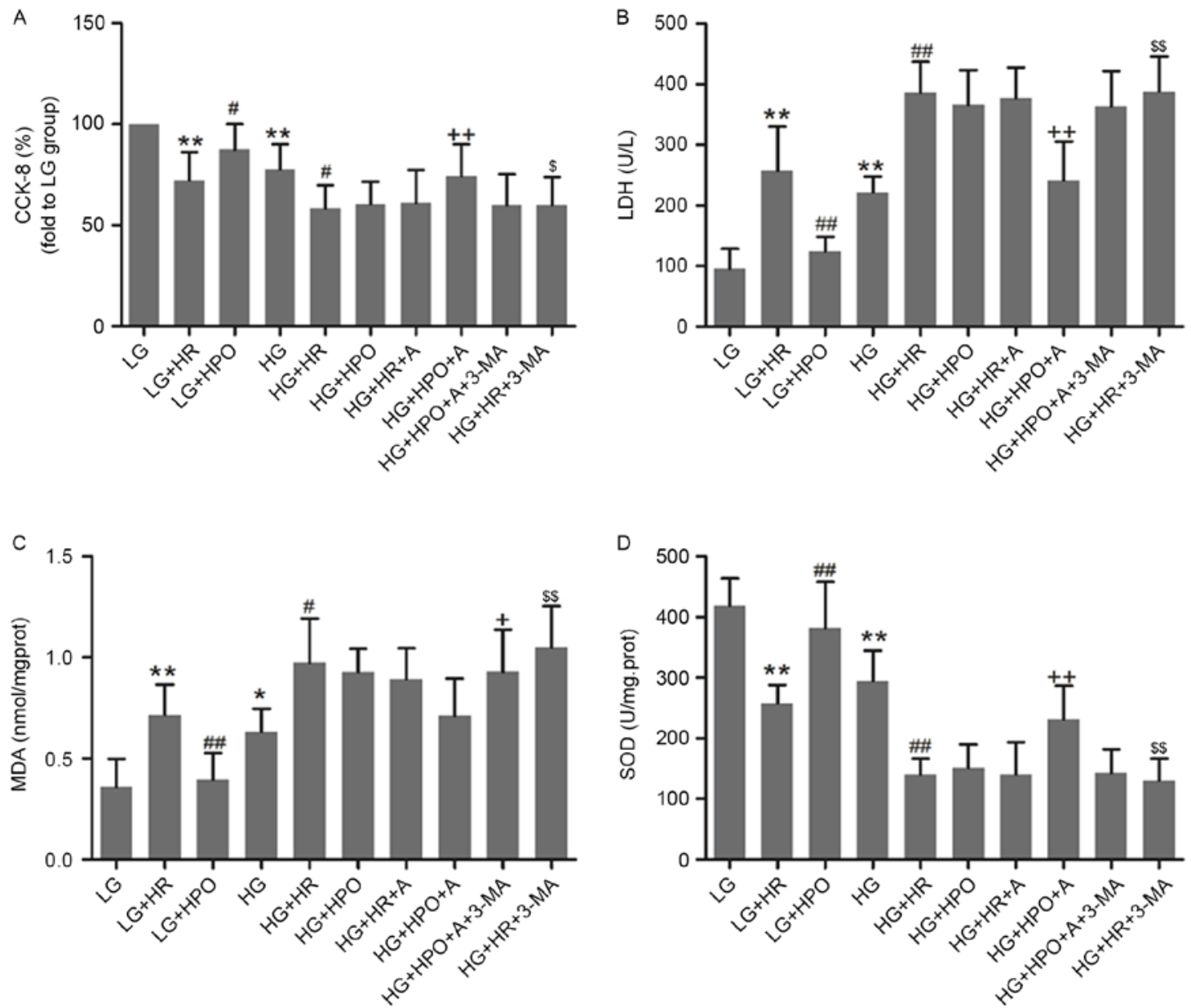

Figure 3. Effects of HPO, with or without A, on H9c2 cells cultured in HG or LG conditions. (A) Results of CCK-8 assay. The expression of (B) LDH, (C) MDA and (D) SOD was analyzed. The results are representative of $\geq 3$ independent experiments. ${ }^{*} \mathrm{P}<0.05$ and ${ }^{* *} \mathrm{P}<0.01$ vs. LG group; ${ }^{*} \mathrm{P}<0.05$ and ${ }^{\# \#} \mathrm{P}<0.01$ vs. LG+HR group; ${ }^{+} \mathrm{P}<0.05$ and ${ }^{++} \mathrm{P}<0.01$ vs. $\mathrm{HG}+\mathrm{HR}$ group; and ${ }^{\$} \mathrm{P}<0.05$ and ${ }^{\$ S} \mathrm{P}<0.01$ vs. HG+HPO+A group. LG, low glucose medium; HG, high glucose medium; HR, hypoxia reoxygenation; HPO, hypoxia post-conditioning; A, A-769662; CCK-8, Cell Counting Kit-8; LDH, lactate dehydrogenase; MDA, malondialdehyde; SOD, superoxide dismutase.

and AMPK phosphorylation, and decreased p62 expression and mTOR phosphorylation. However, these alterations were reversed by the autophagy inhibitor 3-MA.

Effects of HG on HPO cardioprotection, autophagy and the AMPK/mTOR signaling pathway in $\mathrm{H} 9 \mathrm{c} 2$ cell. In vitro, $\mathrm{H} 9 \mathrm{c} 2$ cells were exposed to $\mathrm{HG}$ conditions for $48 \mathrm{~h}$ to simulate the diabetic myocardium. As presented in Fig. 3, HG insult led to decreased cell viability and SOD activity, and increased LDH and MDA release. These alterations were further increased by HR in the LG and HG groups. HPO significantly increased cell viability and SOD activity, and decreased LDH and MDA release in LG medium cultured cells only. As presented in Fig. 4, a decreased LC3B/A ratio and decreased phosphorylated AMPK expression, with increased p62 expression and phosphorylated mTOR expression, was detected in H9c2 cells exposed to HG, compared with the LG group. Following HR insult, the autophagy level and activity of the AMPK/mTOR signaling pathway were upregulated in the LG group, and were further increased by HPO. In the HG groups, HR and HPO did not significantly affect the autophagy level and the AMPK/mTOR signaling pathway.
AMPK activation with A-769662 restores the protective effects of HPO in HG-exposed H9c2 cells. In order to confirm whether AMPK activation restores the protective effects of $\mathrm{HPO}$ in $\mathrm{H} 9 \mathrm{c} 2$ cell lines exposed to $\mathrm{HG}$, cells were pretreated with the AMPK agonist A-769662. As presented in Fig. 3, A-769662 or HPO alone did not confer protective effects to combat HR injury in cells exposed to $\mathrm{HG}$ conditions. By contrast, A-769662 with HPO protected H9c2 cells exposed to $\mathrm{HG}$ conditions from HR injury, as evidenced by increased CCK- 8 and SOD activity, and reduced LDH and MDA release. All of the observed protective effects were reversed by treatment with 3-MA.

Effects of HPO with AMPK activation on autophagy in $H G$-exposed H9c2 cells. As presented in Fig. 4, pretreatment with HPO or A-769662 alone did not affect autophagy status or the AMPK/mTOR signaling pathway. However, HPO with A-769662 significantly activated AMPK/mTOR-regulated autophagy, as evidenced by an increased LC3B/A ratio and increased AMPK phosphorylation, in combination with decreased p62 expression and m-TOR phosphorylation. All of these alterations were reversed by treatment with the 

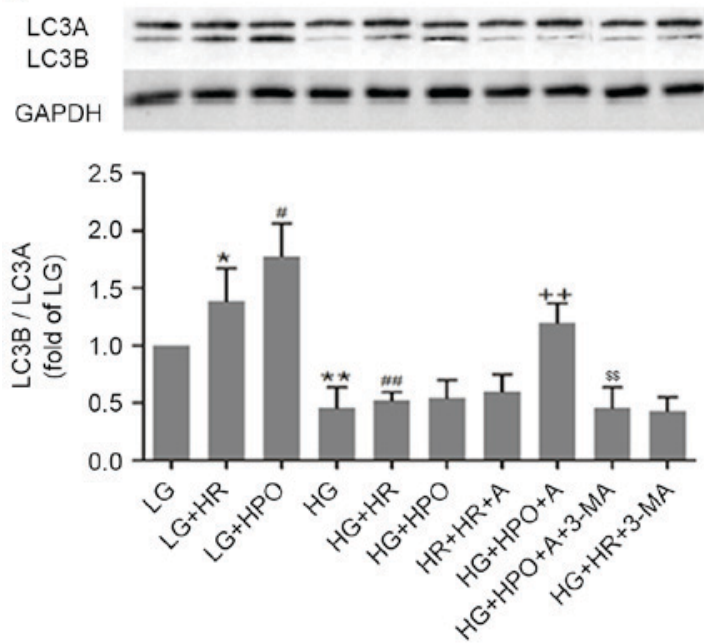

C
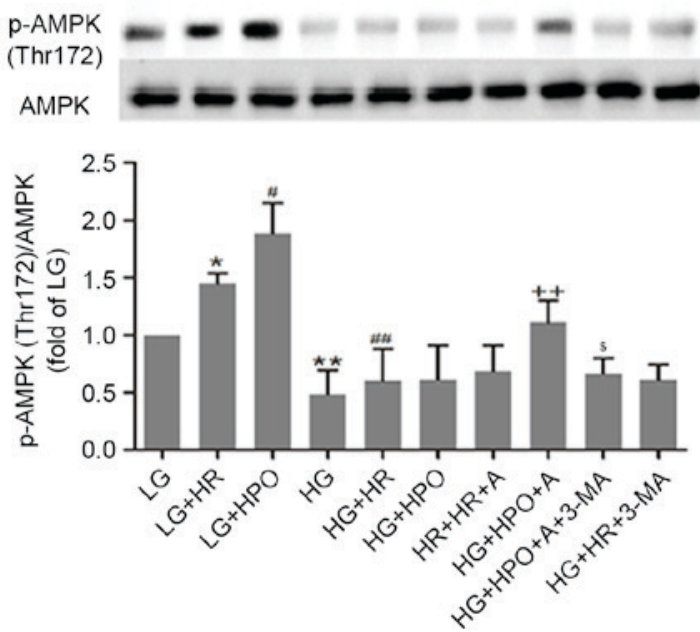

p62
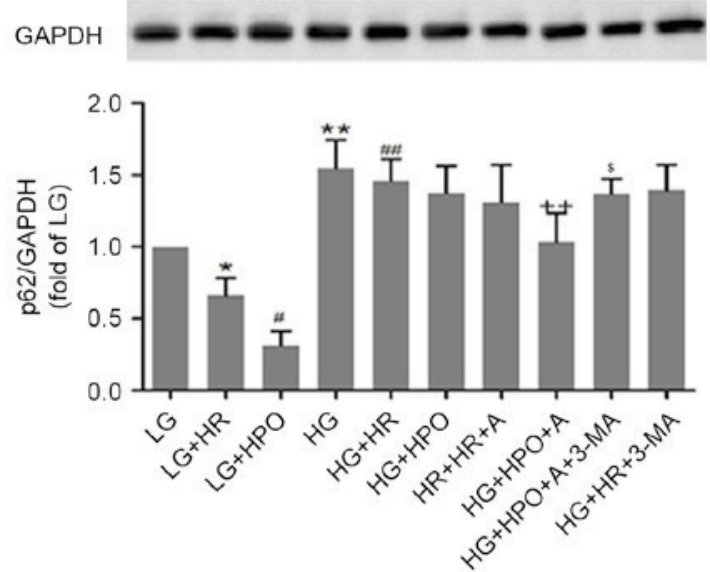

D

p-mTOR

(Ser2448)

mTOR
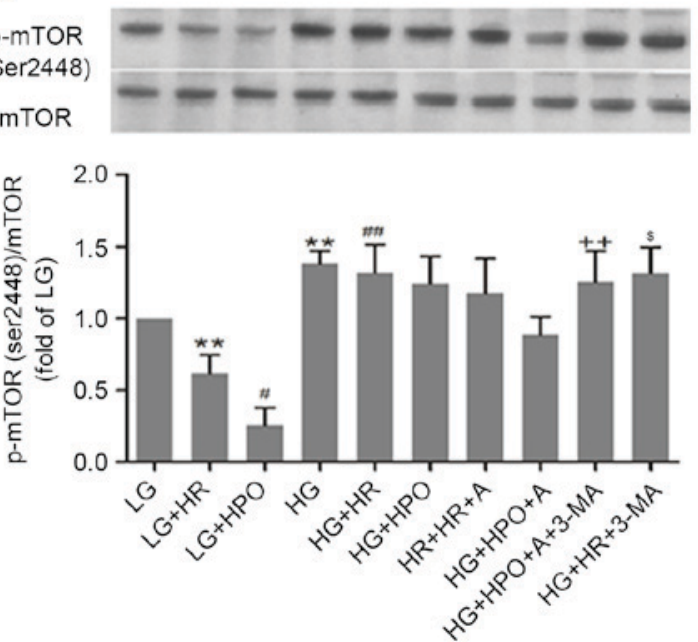

Figure 4. Effects of HPO, with or without A, on autophagy status, and AMPK and mTOR expression and phosphorylation, in H9c2 cells treated with $4 \mathrm{~h}$ of hypoxia followed by $2 \mathrm{~h}$ of reoxygenation. Analysis of (A) LC3B/A ratio, (B) expression of p62, (C) AMPK phosphorylation and (D) mTOR phosphorylation. The results are representative of $\geq 3$ independent experiments. ${ }^{*} \mathrm{P}<0.05$ and ${ }^{* *} \mathrm{P}<0.01$ vs $\mathrm{LG}$ group; ${ }^{*} \mathrm{P}<0.05$ and ${ }^{\# \#} \mathrm{P}<0.01$ vs $\mathrm{LG}+\mathrm{HR}$ group; and ${ }^{++} \mathrm{P}<0.05$ vs. LG+HPO group; ${ }^{\$} \mathrm{P}<0.05$ and ${ }^{\$ \$} \mathrm{P}<0.01$ vs $\mathrm{HG}+\mathrm{HPO}+\mathrm{A}$ group. LG, low glucose medium; HG, high glucose medium; HR, hypoxia reoxygenation; HPO, hypoxia post-conditioning; A, A-769662; LC3, microtubule associated protein 1 light chain 3; AMPK, 5'-AMP-activated protein kinase catalytic subunit $\alpha-1$; mTOR, mammalian target of rapamycin; p62, nuclear pore glycoprotein p62; p, phosphorylated.

autophagy inhibitor 3-MA, which demonstrated that A-769663 and HPO confer their combined protective effects by activating AMPK-regulated autophagy.

\section{Discussion}

The present study demonstrated that hyperglycemia-induced AMPK downregulation contributed to the ineffectiveness of IPO cardioprotection, and that the underlying mechanism may involve myocardial autophagy inhibition. AMPK activation by A-769662 restored the sensitivity of the diabetic myocardium to IPO, possibly by improving autophagy status. To the best of our knowledge, the present study was the first to investigate the effects of AMPK inhibition on IPO cardioprotection in hyperglycemic cardiomyocytes, and the roles of AMPK/mTOR-regulated autophagy in this pathophysiological process.

IPO is an approach whereby brief cycles of ischemia-reperfusion are applied directly following the continued occlusion of a coronary artery, which was first proposed by Zhao et al (2) in 2003. IPO has been demonstrated to be an effective way to relieve myocardial IR injury in animal $(4,5)$ and clinical trials $(25,26)$, and the underlying mechanisms are associated with activation of the reperfusion injury salvage kinase pathway and the Janus kinase/signal transducer and activator of transcription pathway, by inhibiting mitochondrial permeability transition pore opening and antioxidation $(27,28)$. However, according to a number of studies $(4,5,7)$, IPO appears to be unable to induce cardioprotection in diabetes, due in part to severe oxidative stress. Therefore, the present study aimed to investigate the in-depth mechanisms associated with the inefficiency of IPO in diabetes.

Autophagy is an important mechanism in cellular metabolism and survival; it is a dynamic process, which is comprised of autophagosome formation and autolysosomal clearance. Under physiological conditions, a baseline level of autophagy is required to maintain cardiac homeostasis, and autophagy may be activated in response to stress (13). 
However, excessive autophagy results in programmed cell death (29). The conversion of LC3A to LC3B is a marker of autophagosome formation, and an increased ratio of LC3B/A demonstrates an increase in autophagy and a decrease in autolysosome degradation. p62/sequestome 1 , a protein adaptor which is able to bind ubiquitinated cargo designated for autophagic breakdown, was observed to reflect myocardial autophagy status; it is an improved marker of autophagic flux for measuring LC3B/LC3A ratio and levels of p62 (30). Previous studies have demonstrated that autophagy was involved in the pathological process of IR injury. Autophagy was reported to be elevated during ischemia, although whether this is beneficial or detrimental to target organs remains controversial. Huang et al (31) demonstrated that myocardial autophagy inhibition mediated by berberine leads to a decrease in IR-induced myocardium infarct size and cardiac dysfunction, and similar conclusions were drawn in a brain research study by Gao et al (32). By contrast, upregulation of autophagy has been demonstrated to be a potential method of protection against IR injury. Buss et al (10) and Wei et al (22) demonstrated that autophagy activation mitigated IR injury. Zhao et al (14) reported that autophagy mediated by acetylcholine attenuated HR injury in $\mathrm{H} 9 \mathrm{c} 2$ cells, evidenced by increased cell viability and decreased apoptosis. The results of the present study demonstrated that myocardial autophagy was significantly increased following IR insult in the non-diabetic heart, which was increased further following treatment with IPO. However, autophagy inactivation was observed in the diabetic heart, which was consistent with a previous study (20). In addition, as an endogenous protection strategy, autophagic responses failed to be activated by IR or IPO in diabetic hearts; therefore, it was hypothesized that the ineffectiveness of IPO is associated with the inactivation of autophagy in diabetes.

AMPK is a heterotrimeric complex which consists of a catalytic $\alpha$ subunit and two regulatory subunits, $\beta$ and $\gamma$. The serine/threonine kinase activity of AMPK is mediated by the $\alpha$ subunit, and is characterized by the presence of a threonine residue (Thr172) in a loop that must be phosphorylated for activation to occur (33). AMPK protein is expressed in the majority of mammalian tissues, including those of the cardiovascular system; it is a highly-conserved sensor of the cellular energy status and serves a role in regulating cellular biological activity. mTOR, an additional highly-conserved serine/threonine protein kinase, is important for cell growth, proliferation and differentiation. AMPK has been demonstrated to be an upstream protein, which is able to negatively regulate $\mathrm{mTOR}$ in a directly or indirect manner (12). Previous studies have indicated that the AMPK/mTOR signaling pathway is associated with autophagy regulation, which serves a role in the occurrence and development of a number of diseases. Guo et al (19) and Zhao et al (14) observed that an IR insult upregulated AMPK phosphorylation and downregulated mTOR phosphorylation, with an increased level of autophagy in cardiomyocytes in vivo and in vitro. Consistent with the previous studies mentioned above, the results of the present study demonstrated that AMPK/mTOR pathway activity was promoted by IR insult in the non-diabetic myocardium, and that IPO further activated the AMPK/mTOR pathway, in combination with an increased level of autophagy. However, in the diabetic myocardium, phosphorylation of AMPK was inhibited, which was consistent with studies by Guo et al (20) and Viollet et al (34). In addition, the present study demonstrated that IR and IPO were unable to activate the AMPK/mTOR signaling pathway efficiently in diabetes.

In order to confirm whether hyperglycemia-induced AMPK inhibition contributes to the ineffectiveness of IPO cardioprotection by decreasing myocardial autophagy, the AMPK agonist 769662 and the autophagy inhibitor 3-MA were applied in vivo and in vitro. A-769662 was observed to activate AMPK efficiently by allosteric inhibition of AMPK dephosphorylation at the Thr172 site, in a previous study (35). An additional previous study demonstrated that A-769662 did not affect the total expression of AMPK, although it significantly increased the phosphorylation of AMPK at Thr172. AMPK activation by A-769662 was reported to exert cardioprotection by increasing the expression level of a downstream signaling pathway involving endothelial NO synthase, thereby stimulating NO release (21). Kim et al (36) demonstrated that pretreatment with A-769662 in vivo decreased infarct size in $\mathrm{C} 57 \mathrm{Bl} / 6$ mice undergoing left coronary artery occlusion and reperfusion. Similarly, Paiva et al (37) demonstrated that directly enhancing AMPK activation with A-769662 at reperfusion protects the IR rat myocardium against infarction. Notably, all of the above previous studies were performed in non-diabetic conditions. In the present study, A-769662 nor IPO alone did not attenuate IR injury in diabetic hearts. By contrast, A-769662 administration in combination with IPO treatment significantly protected diabetic hearts from IR injury, with a simultaneous increase in autophagy being observed. In addition, it was observed that the protection mediated by A-762669 with IPO was reversed by the autophagy inhibitor 3-MA, with a decrease in the myocardial autophagy level, which further demonstrated that autophagy is associated with the protective mechanism of IPO. The results obtained from cultured H9c2 cells in the present study were consistent with the in vivo experiments.

In conclusion, the present study confirmed the ineffectiveness of IPO cardioprotection in diabetes, and demonstrated that hyperglycemia-induced AMPK inhibition underlies this ineffectiveness, in part by decreased myocardial autophagy. AMPK activation mediated by A-769662 restored the sensitivity of diabetic hearts to IPO cardioprotection, through autophagy activation. Therefore, the present study demonstrated that targeting AMPK may elicit IPO cardioprotection in human diabetes.

\section{References}

1. Beckman JA, Paneni F, Cosentino F and Creager MA: Diabetes and vascular disease: Pathophysiology, clinical consequences, and medical therapy: Part II. Eur Heart J 34: 2444-2452, 2013.

2. Zhao ZQ, Corvera JS, Halkos ME, Kerendi F, Wang NP, Guyton RA and Vinten-Johansen J: Inhibition of myocardial injury by ischemic postconditioning during reperfusion: Comparison with ischemic preconditioning. Am J Physiol Heart Circ Physiol 285: H579-H588, 2003.

3. Bochaton T, Crola-Da-Silva C, Pillot B, Villedieu C, Ferreras L, Alam MR, Thibault H, Strina M, Gharib A, Ovize M and Baetz D: Inhibition of myocardial reperfusion injury by ischemic postconditioning requires sirtuin 3-mediated deacetylation of cyclophilin D. J Mol Cell Cardiol 84: 61-69, 2015. 
4. Xue R, Lei S, Xia ZY, Wu Y, Meng Q, Zhan L, Su W, Liu H, Xu J, Liu Z, et al: Selective inhibition of PTEN preserves ischaemic post-conditioning cardioprotection in STZ-induced Type 1 diabetic rats: Role of the PI3K/Akt and JAK2/STAT3 pathways. Clin Sci (Lond) 130: 377-392, 2016.

5. Liu M, Zhou B, Xia ZY, Zhao B, Lei SQ, Yang QJ, Xue R, Leng Y, $\mathrm{Xu} \mathrm{JJ}$ and Xia Z: Hyperglycemia-induced inhibition of DJ-1 expression compromised the effectiveness of ischemic postconditioning cardioprotection in rats. Oxid Med Cell Longev 2013 $564902,2013$.

6. Drenger B, Ostrovsky IA, Barak M, Nechemia-Arbely Y, Ziv E and Axelrod JH: Diabetes blockade of sevoflurane postconditioning is not restored by insulin in the rat heart: Phosphorylated signal transducer and activator of transcription 3- and phosphatidylinositol 3-kinase-mediated inhibition. Anesthesiology 114: 1364-1372, 2011

7. Przyklenk K, Maynard M, Greiner DL and Whittaker P: Cardioprotection with postconditioning: Loss of efficacy in murine models of type-2 and type-1 diabetes. Antioxid Redox Signal 14: 781-790, 2011.

8. Mihaylova MM and Shaw RJ: The AMPK signalling pathway coordinates cell growth, autophagy and metabolism. Nat Cell Biol 13: 1016-1023, 2011.

9. Jeon SM, Chandel NS and Hay N: AMPK regulates NADPH homeostasis to promote tumour cell survival during energy stress. Nature 485: 661-665, 2012.

10. Buss SJ, Riffel JH, Katus HA and Hardt SE: Augmentation of autophagy by mTOR-inhibition in myocardial infarction: When size matters. Autophagy 6: 304-306, 2010.

11. She C, Zhu LQ, Zhen YF, Wang XD and Dong QR: Activation of AMPK protects against hydrogen peroxide-induced osteoblast apoptosis through autophagy induction and NADPH main tenance: New implications for osteonecrosis treatment? Cell Signal 26: 1-8, 2014

12. Kim J, Kundu M, Viollet B and Guan KL: AMPK and mTOR regulate autophagy through direct phosphorylation of Ulk1. Nat Cell Biol 13: 132-141, 2011.

13. Mizushima N, Levine B, Cuervo AM and Klionsky DJ: Autophagy fights disease through cellular self-digestion. Nature 451: 1069-1075, 2008

14. Zhao M, Sun L, Yu XJ, Miao Y, Liu JJ, Wang H, Ren J and Zang WJ: Acetylcholine mediates AMPK-dependent autophagic cytoprotection in $\mathrm{H} 9 \mathrm{c} 2$ cells during hypoxia/reoxygenation injury. Cell Physiol Biochem 32: 601-613, 2013.

15. Jiang T, Yu JT, Zhu XC, Zhang QQ, Tan MS, Cao L, Wang HF, Shi JQ, Gao L, Qin H, et al: Ischemic preconditioning provides neuroprotection by induction of AMP-activated protein kinase-dependent autophagy in a rat model of ischemic stroke. Mol Neurobiol 51: 220-229, 2015.

16. Nepal S and Park PH: Activation of autophagy by globular adiponectin attenuates ethanol-induced apoptosis in HepG2 cells: Involvement of AMPK/FoxO3A axis. Biochim Biophys Acta 1833: 2111-2125, 2013

17. Wang LT, Chen BL, Wu CT, Huang KH, Chiang CK and Hwa Liu S: Protective role of AMP-activated protein kinase-evoked autophagy on an in vitro model of ischemia/reperfusion-induced renal tubular cell injury. PLoS One 8: e79814, 2013.

18. Pauly M, Daussin F, Burelle Y, Li T, Godin R, Fauconnier J, Koechlin-Ramonatxo C, Hugon G, Lacampagne A, Coisy-Quivy M, et al: AMPK activation stimulates autophagy and ameliorates muscular dystrophy in the $\mathrm{mdx}$ mouse diaphragm. Am J Pathol 181: 583-592, 2012.

19. Guo L, Xu JM and Mo XY: Ischemic postconditioning regulates cardiomyocyte autophagic activity following ischemia/reperfusion injury. Mol Med Rep 12: 1169-1176, 2015.

20. Guo Y, Yu W, Sun D, Wang J, Li C, Zhang R, Babcock SA, Li Y, Liu M, Ma M, et al: A novel protective mechanism for mitochondrial aldehyde dehydrogenase (ALDH2) in type i diabetes-induced cardiac dysfunction: Role of AMPK-regulated autophagy. Biochim Biophys Acta 1852: 319-331, 2015.

21. Song T, Lv LY, Xu J, Tian ZY, Cui WY, Wang QS, Qu G and Shi XM: Diet-induced obesity suppresses sevoflurane preconditioning against myocardial ischemia-reperfusion injury: Role of AMP-activated protein kinase pathway. Exp Biol Med (Maywood) 236: 1427-1436, 2011.
22. Wei C, Li H, Han L, Zhang L and Yang X: Activation of autophagy in ischemic postconditioning contributes to cardioprotective effects against ischemia/reperfusion injury in rat hearts. J Cardiovasc Pharmacol 61: 416-422, 2013.

23. Swanlund JM, Kregel KC and Oberley TD: Investigating autophagy: Quantitative morphometric analysis using electron microscopy. Autophagy 6: 270-277, 2010.

24. Wang W, Yan J, Wang H, Shi M, Zhang M, Yang W, Peng C and $\mathrm{Li} \mathrm{H}$ : Rapamycin ameliorates inflammation and fibrosis in the early phase of cirrhotic portal hypertension in rats through inhibition of mTORC1 but not mTORC2. PLoS One 9: e83908, 2014

25. Luo W, Zhu M, Huang R and Zhang Y: A comparison of cardiac post-conditioning and remote pre-conditioning in paediatric cardiac surgery. Cardiol Young 21: 266-270, 2011.

26. Durdu S, Sirlak M, Cetintas D, Inan MB, Eryılmaz S, Ozcinar E, Yazicioglu L, Elhan AH, Akar AR and Uysalel A: The efficacies of modified mechanical post conditioning on myocardial protection for patients undergoing coronary artery bypass grafting. J Cardiothorac Surg 7: 73, 2012.

27. Hausenloy DJ: Signalling pathways in ischaemic postconditioning. Thromb Haemost 101: 626-634, 2009.

28. Ovize M, Baxter GF, Di Lisa F, Ferdinandy P, Garcia-Dorado D, Hausenloy DJ, Heusch G, Vinten-Johansen J, Yellon DM and Schulz R; Working Group of Cellular Biology of Heart of European Society of Cardiology: Postconditioning and protection from reperfusion injury: Where do we stand? Position paper from the working group of cellular biology of the heart of the european society of cardiology. Cardiovasc Res 87: 406-423, 2010.

29. Hariharan N, Zhai P and Sadoshima J: Oxidative stress stimulates autophagic flux during ischemia/reperfusion. Antioxid Redox Signal 14: 2179-2190, 2011.

30. Klionsky DJ, Abdalla FC, Abeliovich H, Abraham RT, Acevedo-Arozena A, Adeli K, Agholme L, Agnello M, Agostinis P, Aguirre-Ghiso JA, et al: Guidelines for the use and interpretation of assays for monitoring autophagy. Autophagy 8: 445-544, 2012

31. Huang Z, Han Z, Ye B, Dai Z, Shan P, Lu Z, Dai K, Wang C and Huang W: Berberine alleviates cardiac ischemia/reperfusion injury by inhibiting excessive autophagy in cardiomyocytes. Eur J Pharmacol 762: 1-10, 2015.

32. Gao L, Jiang T, Guo J, Liu Y, Cui G, Gu L, Su L and Zhang Y: Inhibition of autophagy contributes to ischemic postconditioning-induced neuroprotection against focal cerebral ischemia in rats. PLoS One 7: e46092, 2012.

33. Hawley SA, Davison M, Woods A, Davies SP, Beri RK, Carling D and Hardie DG: Characterization of the AMP-activated protein kinase kinase from rat liver and identification of threonine 172 as the major site at which it phosphorylates AMP-activated protein kinase. J Biol Chem 271: 27879-27887, 1996.

34. Viollet B, Lantier L, Devin-Leclerc J, Hebrard S, Amouyal C, Mounier R, Foretz M and Andreelli F: Targeting the AMPK pathway for the treatment of Type 2 diabetes. Front Biosci (Landmark Ed) 14: 3380-3400, 2009.

35. Sanders MJ, Ali ZS, Hegarty BD, Heath R, Snowden MA and Carling D: Defining the mechanism of activation of AMP-activated protein kinase by the small molecule A-769662, a member of the thienopyridone family. J Biol Chem 282: 32539-32548, 2007.

36. Kim AS, Miller EJ, Wright TM, Li J, Qi D, Atsina K, Zaha V, Sakamoto K and Young LH: A small molecule AMPK activator protects the heart against ischemia-reperfusion injury. J Mol Cell Cardiol 51: 24-32, 2011.

37. Paiva MA, Goncalves LM, Providência LA, Davidson SM, Yellon DM and Mocanu MM: Transitory activation of AMPK at reperfusion protects the ischaemic-reperfused rat myocardium against infarction. Cardiovasc Drugs Ther 24: 25-32, 2010. 